\title{
Equações brasileiras: força muscular inspiratória
}

\author{
Brazilian equations: inspiratory muscle force \\ Ecuaciones brasileñas: fuerza muscular inspiratoria \\ Isabela Maria Braga Sclauser Pessoa', Débora Alves Pinheiro², Myrna Amaral Dias Falcão³, \\ Paloma Ferreira de Araújo ${ }^{4}$, Patrícia Marília Martins ${ }^{5}$, Marcelo Velloso ${ }^{6}$
}

\begin{abstract}
RESUMO I A força dos músculos respiratórios (FMR) pressão inspiratória máxima [PImáx] e pressão expiratória máxima [PEmáx] - apresentam variação quando estimadas por equações de predição. O objetivo deste estudo foi verificar se a classificação da PImáx obtida pelas equações de predição propostas por autores brasileiros é semelhante e concordante. A amostra foi constituída por 18 pacientes estáveis com disfunções cardiorrespiratória, neurológica e fraqueza muscular respiratória. A PImáx foi medida pelo manovacuômetro analógico e comparada com as equações de predição de autores brasileiros. Apenas dois autores detectaram fraqueza muscular inspiratória $(p<0,0001)$. Ao avaliar a concordância entre autores (Bias), verificou-se baixa concordância entre os valores preditos pelas equações, exceto entre os autores que detectaram fraqueza muscular inspiratória nos pacientes avaliados.

Descritores | Valores de Referência; Força Muscular; Músculos Respiratórios.
\end{abstract}

ABSTRACT | Respiratory muscle strength (RMS) (maximal inspiratory pressure [MIP] and maximal expiratory pressure [MEP]) vary when estimated by prediction equations. This study will verify whether the classification of MIP obtained by the prediction equations proposed by Brazilian authors is similar and concordant. The sample consisted of 18 stable patients with cardiorespiratory and neurological dysfunctions and respiratory muscle weakness. The MIP was measured by the analog compound gauge and compared to the prediction equations of Brazilian authors. Only two authors found inspiratory muscle weakness ( $p<0.0001)$. Assessing the agreement among authors (BIAS), there was a low agreement between the values predicted by the equations, except among the authors who detected inspiratory muscle weakness in the evaluated patients.

Keywords I Reference Values; Muscle Strength; Respiratory Muscles.

RESUMEN | La fuerza muscular respiratoria (MRF) (presión inspiratoria máxima [MMAX] y presión espiratoria máxima [Mepmax]) varían cuando se estima por ecuaciones de predicción. El objetivo de este estudio era verificar si la clasificación del MIP obtenida por las ecuaciones de predicción propuestas por los autores brasileños es similar y concordante. La muestra consistió en 18 pacientes estables con debilidad cardiorrespiratoria, neurológica y muscular respiratoria. Mmax fue medido por el manovacuómetro analógico y comparado con las ecuaciones de predicción de autores brasileños. Solo dos autores debilidad muscular inspiradora $(p<0.0001)$. Al evaluar la concordancia entre los autores (Bias), hubo una baja concordancia entre los valores predichos por las ecuaciones a excepción de los autores que detectaron debilidad de los músculos inspiratorios en los pacientes evaluados.

Palabras Clave I Valores de Referencia; Fuerza Muscular; Músculos Respiratorios.

Estudo realizado na Clínica escola da Pontifícia Universidade Católica de Minas Gerais, Betim, Minas Gerais, Brasil.

PPontifícia Universidade Católica de Minas Gerais (PUC) - Belo Horizonte (MG), Brasil. E-mail: isa.sclauser@terra.com.br.

Orcid: 0000-0002-2352-8954

2Pontifícia Universidade Católica de Minas Gerais (PUC) - Belo Horizonte (MG), Brasil. E-mail: deborafap@hotmail.com.

Orcid: 0000-0002-8986-7799

${ }_{3}^{3}$ Pontifícia Universidade Católica de Minas Gerais (PUC) - Belo Horizonte (MG), Brasil. E-mail: myrnaadf@gmail.com.

Orcid: 0000-0001-6222-0293

4Pontifícia Universidade Católica de Minas Gerais (PUC) - Belo Horizonte (MG), Brasil. E-mail: palomafisio.ferreira@gmail.com. Orcid: 0000-0003-4611-871X

${ }^{5}$ Pontifícia Universidade Católica de Minas Gerais (PUC) - Belo Horizonte (MG), Brasil. E-mail: patriciamartinsfisio@hotmail.com. Orcid: 0000-0002-5840-4767

${ }^{6}$ Universidade Federal de Minas Gerais (UFMG) - Belo Horizonte (MG), Brasil. E-mail: marcello.vel@gmail.com. Orcid: 0000-0001-9361-8149 


\section{INTRODUÇÃO}

A força dos músculos respiratórios (FMR) é refletida pela pressão desenvolvida por eles (pressão motriz do sistema respiratório), que comanda a ventilação e é registrada como pressão respiratória máxima $(\mathrm{PRM})^{1}$. A manobra clássica de avaliação da força muscular inspiratória é aquela em que o sujeito gera esforço inspiratório estático máximo, ou seja: pressão inspiratória máxima (PImáx) contra uma peça bocal ocluída ${ }^{1,2}$.

A partir da década de 1960, vários autores ${ }^{3-5}$ avaliaram a PImáx em indivíduos de diferentes países, de faixas etárias distintas e estabeleceram valores de referência. Esses valores foram relatados como média e desvio-padrão ou mediana e, em alguns estudos, foram propostas equações de predição. A literatura, porém, mostra grande variação entre os valores preditos para a PImáx ${ }^{4,5}$.

Sabe-se que o treinamento muscular inspiratório em indivíduos com disfunções pulmonares, cardíacas e neurológicas, maximiza a reserva ventilatória, melhora os sintomas de dispneia e a eficácia da tosse ${ }^{6,7}$ Segundo a American Thoracic Society/European Respiratory Society (ATS/ERS) ${ }^{1}$ e a Sociedade Brasileira de Pneumologia e Tisiologia $(\mathrm{SBPT})^{2}$, valores de PImáx menores do que $-80 \mathrm{cmH}_{2} \mathrm{O}$ permitem afastar a presença de fraqueza muscular ventilatória. Dessa forma, a classificação correta da PImáx, a partir do valor predito, se faz necessária para elaborar o plano de tratamento fisioterapêutico adequado.

No Brasil, quatro grupos de pesquisadores ${ }^{5,8-10}$ propuseram equações de predição para as PRM. Tendo em vista a ausência de estudos, por parte de pesquisadores brasileiros, que comparam as equações de predição e também os valores obtidos e preditos por esses autores em uma amostra de indivíduos com fraqueza muscular respiratória, este estudo teve como objetivo verificar se a classificação da PImáx obtida pelas equações de predição propostas por autores brasileiros é semelhante e concordante.

\section{METODOLOGIA}

Trata-se de um estudo observacional, descritivo, do tipo transversal. O estudo foi aprovado pelo Comitê de Ética em Pesquisa em Seres Humanos (CEP) da Pontifícia Universidade Católica de Minas Gerais (PUC-Minas), CAEE no 56300416.1.0000.5137, e todos os participantes assinaram o termo de consentimento livre e esclarecido. A amostra foi de conveniência e os indivíduos foram selecionados da Clínica Escola da PUC-Minas no período de agosto a dezembro de 2016.

Os critérios de inclusão foram: indivíduos com disfunções pulmonares, cardíacas e neurológicas com valor de $\mathrm{PImáx}>-80 \mathrm{cmH}_{2} \mathrm{O}$; sem déficit cognitivo, avaliado pelo Mini Exame do Estado Mental (MEEM); que não apresentassem contraindicações à realização das PRM, segundo a Sociedade Brasileira de Pneumologia e Tisiologia (SBPT) ${ }^{2}$, estáveis hemodinamicamente, com pressão arterial (PA) em repouso menor que 160/110mmHg; saturação periférica de hemoglobina em oxigênio $\left(\mathrm{SpO}_{2}\right)$ maior que $88 \%$ e frequência cardíaca (FC) menor que $100 \mathrm{bpm}$. Foram excluídos os indivíduos com incapacidade de executar os procedimentos do protocolo de pesquisa.

No primeiro dia, os indivíduos foram submetidos a uma anamnese, incluindo a coleta de dados vitais: PA (TYCOS ${ }^{\circledR}$, São Paulo, Brasil), FC e $\mathrm{SpO}_{2}$ (NONIN ${ }^{\circledR}$, modelo 9500, Plymouth (MN), EUA) e dados antropométricos como: circunferência abdominal e relação cintura-quadril (ICQ), utilizando uma fita métrica de plástico inelástica ${ }^{11}$, peso (TANITA ${ }^{\circledR}$, modelo BC-543, Londres, Reino Unido), altura (Standard Sanny ${ }^{\circledR}$, modelo ES2030, São Paulo, Brasil) e índice de massa corporal (IMC) ${ }^{12}$ obtido a partir da divisão do peso $(\mathrm{kg})$ pela altura ${ }^{2}\left(\mathrm{~cm}^{2}\right)^{11}$. Os indivíduos acima de 60 anos foram submetidos à avaliação da cognição por meio do questionário $\mathrm{MEEM}^{13}$. Com a finalidade de caracterização da amostra, foram considerados o diagnóstico médico dos indivíduos mediante Classificação Estatística Internacional de Doenças e Problemas Relacionados com a Saúde (CID).

No segundo encontro, foi mensurada a PImáx dos indivíduos pelo manovacuômetro (MVC), analógico previamente calibrado (WIKA ${ }^{\circledR}$, modelo Type 213.53, Brasil), graduado em $\mathrm{cmH}_{2} \mathrm{O}$, com variação de intervalo $\pm 150 \mathrm{cmH}_{2} \mathrm{O}$, tubo de silicone, interface/bocal do tipo retangular ${ }^{14}$, com um orifício de fuga de $2 \mathrm{~mm}$ de diâmetro $^{2}$. Todos os indivíduos receberam instruções e demonstração previamente aos testes. Foi preconizado o uso do clipe nasal e a posição sentada, com os pés e o tronco apoiados ${ }^{1,2}$.

A medida da PImáx foi realizada a partir do volume residual $(\mathrm{VR})^{1,2} \mathrm{e}$ todos os participantes realizaram pelo menos cinco manobras com intervalo de um minuto entre elas. A mensuração das pressões era considerada completa quando o indivíduo realizasse três manobras 
aceitáveis e, dentre essas, duas reprodutíveis (duas com variação igual ou inferior a $10 \%$, com a pressão de maior valor $)^{1}$, desde que esse não tenha sido o último teste realizado ${ }^{2}$. O maior valor da PImáx foi selecionado para as análises deste estudo. Os valores medidos para cada indivíduo foram comparados aos propostos por autores brasileiros: Neder et al. ${ }^{8}$, Costa et al. ${ }^{9}$ e Simões et al. ${ }^{10}$ e Pessoa et al. ${ }^{5}$.

Para o cálculo amostral, foi utilizado o programa G*Power 3.1 (Statistical Power Analyses). Como o valor previsto no cálculo amostral foi baixo $(n=7)$, optou-se por analisar o poder do estudo ao final das coletas. Os dados foram apresentados como medidas de tendência central, medidas de variabilidade e percentual. A distribuição das variáveis foi verificada pelo teste de Shapiro-Wilk. A análise de variância (Anova) para medidas repetidas foi utilizada para as comparações dos valores das PImáx preditas pelas equações brasileiras em relação aos valores obtidos pelos indivíduos na avaliação de FMR, seguida pelo teste post-hoc de Bonferroni. O método de BlandAltman foi realizado para avaliar a concordância dos valores preditos, a partir da visualização gráfica da dispersão entre a diferença e a média das variáveis. A análise estatística foi realizada no programa Statistical Package for the Social Science (SPSS 20.0.0) O nível de significância considerado foi de $5 \%(\mathrm{p}<0,05)$.

\section{RESULTADOS}

Foram recrutados 23 indivíduos, com idades entre 20 e 89 anos e de ambos os sexos, dos quais cinco foram excluídos - dois por não atender aos critérios de aceitabilidade e reprodutibilidade do teste da PImáx, dois por não apresentar fraqueza muscular inspiratória e um por não alcançar escore suficiente no MEEM. Assim, a amostra final foi constituída por 18 indivíduos, sendo 6 com cardiopatia, 10 com pneumopatia e 2 com disfunção neurológica (Tabela 1).

Tabela 1. Caracterização antropométrica e clínica da amostra (N=18)

$\begin{array}{lr}\text { Parâmetros } & \begin{array}{r}\text { Indivíduos com disfunções } \\ \text { pulmonar, cardíaca e neurológica }\end{array} \\ \text { Sexo }(\mathrm{F} / \mathrm{M}) & 10 / 8 \\ \text { Idade (anos) } & 57(13,56) \\ \text { Sedentarismo } & 18 \\ \text { IMC }\left(\mathrm{kg} / \mathrm{m}^{2}\right) & 24,08(4,58) \\ \text { ICQ }(\mathrm{cm}) & \mathrm{F}: 0,877 \pm 0,1 / \mathrm{M}: 0,94 \pm 0,1 \\ \text { MEEM (pontos }) & 24,44(3,63) \\ \text { PImáx pico }\left(\mathrm{CmH}_{2} \mathrm{O}\right) & 50,00(16,18), \text { min=25 / máx=78 } \\ \text { PImáx sustentada }\left(\mathrm{CmH}_{2} \mathrm{O}\right) & 48,11(18,83), \text { min=20 / máx=78 } \\ \text { PEmáx pico }\left(\mathrm{CmH}_{2} \mathrm{O}\right) & 50,66(18,92), \text { min=20 / máx=98 } \\ \text { PEmáx sustentada }\left(\mathrm{CmH}_{2} \mathrm{O}\right) & 48,72(19,52), \text { min=20 / máx=98 }\end{array}$

Dados apresentados como média e desvio-padrão (DP); N: número de participantes; F: feminino; M: masculino; IMC: índice de massa corporal; ICQ: indice cintura-quadril; MEEM: Mini Exame do Estado Mental; PIMáx: pressão inspiratória máxima; PEmáx: pressão expiratória máxima; min: valor mínimo; máx: valor máximo.

A Tabela 2 apresenta a comparação entre os valores obtidos pelos pacientes com os preditos para a PImáx. Ao comparar o valor predito para a PImáx pico e sustentada com a média obtida para população, Costa et al. ${ }^{9}$ e Simões et al. ${ }^{10}$ não apresentaram diferença significativa $(p=0,90$ e $\mathrm{p}=0,56$, respectivamente). Observaram-se diferenças significativas entre os resultados das equações de predição entre os autores brasileiros $(\mathrm{p}<0,01)$.

A análise de Bland-Altman mostrou os elevados valores de Bias e a marcante dispersão dos valores preditos pelos diferentes autores brasileiros para a PImáx (Figura 1).

Tabela 2. Comparação entre os valores obtidos pelos pacientes versus valores preditos para a Plmáx pelas equações propostas pelos autores brasileiros $(\mathrm{N}=18)$

\begin{tabular}{|c|c|c|c|c|c|}
\hline Autores & $\begin{array}{l}\text { PImáx } \\
\text { prev. }\end{array}$ & $\begin{array}{l}\text { PImáx pico } \\
\text { Obt. }\end{array}$ & $\begin{array}{l}\text { PImáx sust. } \\
\text { obt. }\end{array}$ & $\begin{array}{c}\text { Dif PImáx pico x obt. } \\
\text { Pvalor }\end{array}$ & PImáx sust. Pvalor \\
\hline Neder et al. & $93,5(3,82)$ & & & 0,00 & 0,00 \\
\hline Costa et al. & $63,01(5,02)$ & $50,0(3,81)$ & $48,11(4,44)$ & 0,56 & 0,35 \\
\hline Simões et al. & $44,74(8,33)$ & & & 1,00 & 1,00 \\
\hline Pessoa et al. & $78,15(4,09)$ & & & 0,00 & 0,00 \\
\hline
\end{tabular}

Dados apresentados como média e desvio-padrão (DP): PImáx prev.: pressão inspiratória máxima predita; PImáx pico obt.: pressão inspiratória máximo pico obtida; PImáx sust. obt.: pressão inspiratória máxima sustentada obtida; Dif PImáx pico × obt.: diferença entre as médias das pressões inspiratórias máximas previstas e obtidas; Pvalor: valor estatístico. 

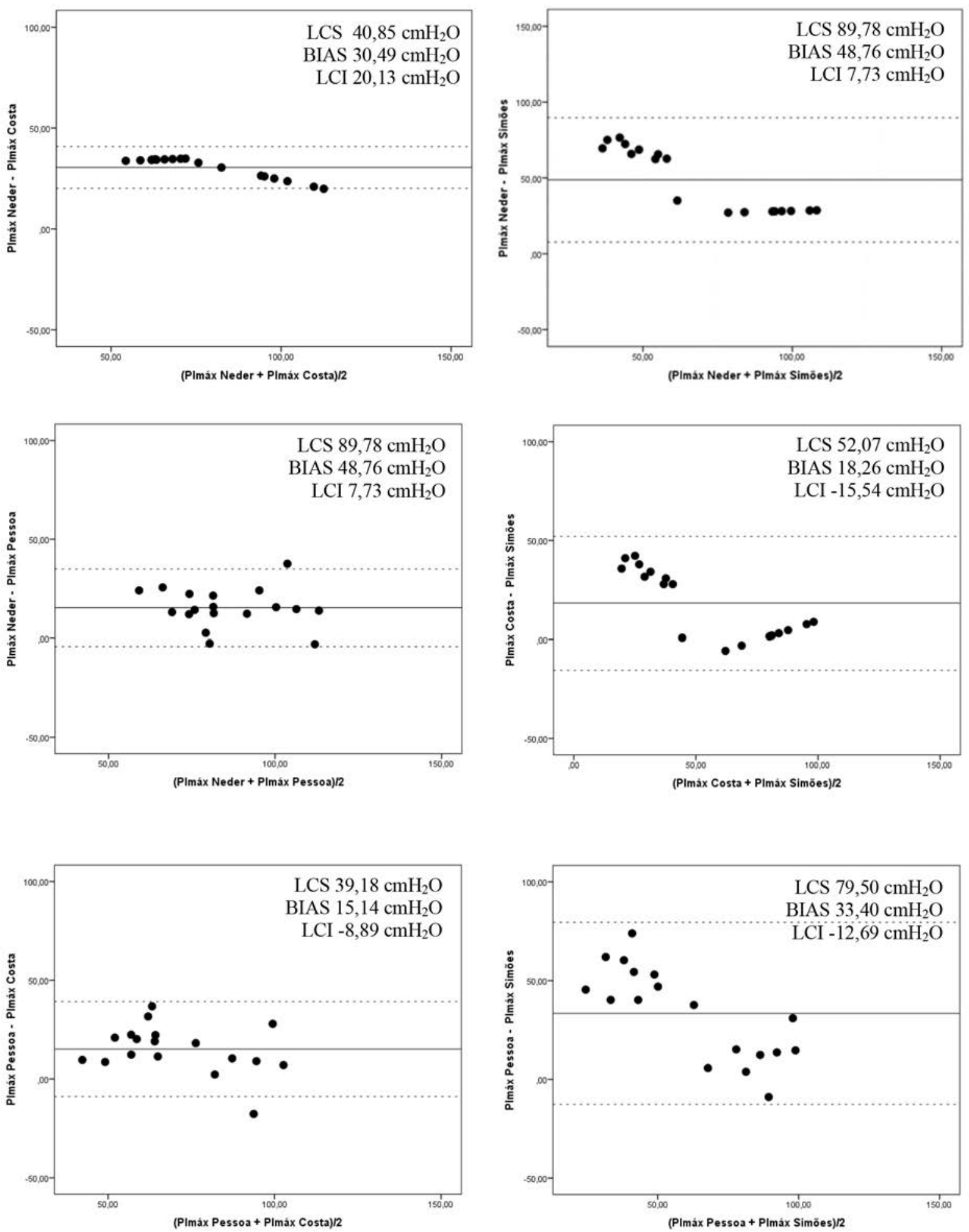

Figura 1. Análise de Bland-Altman entre os valores preditos para as medidas da pressão inspiratória máxima (PImáx). LCS: limite de concordância superior; LCI: limite de concordância inferior.

\section{DISCUSSÃo}

Este estudo foi o primeiro a realizar uma análise comparativa e de concordância entre os resultados das equações brasileiras de predição das PImáx em uma amostra de indivíduos com fraqueza dos músculos respiratórios. Destaca-se que os valores preditos para a PImáx pelas equações de Neder et al. ${ }^{8}$ e Pessoa et al. ${ }^{5}$ apresentaram diferença estatística comparados com os obtidos pelos pacientes, sendo o valor médio predito 
superior ao valor médio obtido, detectando a real fraqueza muscular inspiratória da amostra selecionada. Neder et al. ${ }^{8}$ e Pessoa et al. ${ }^{5}$ apresentaram uma maior concordância entre os resultados das equações de predição.

Até a década de 1950, a grande variabilidade dos valores das PRM entre os estudos realizados provocou o questionamento, por parte dos pesquisadores, sobre a utilidade dos testes ${ }^{3}$. Shephard et al., em 1958, citados por Ringqvist ${ }^{3}$, relataram que a variabilidade dos dados até então registrados na literatura, além de apresentarem base teórica incerta, desvalorizavam os testes das PRM.

A constatação da variação entre os valores preditos pelas equações de predição por diversos autores ${ }^{15-18}$ instigou a publicação, em 2002, das diretrizes internacional - ATS/ERS ${ }^{1-}$ e nacional - $\mathrm{SBPT}^{2}$ - sobre as PRM. Essas diretrizes apresentam recomendações similares, embora haja pontos não concordantes. $\mathrm{Na}$ diretriz internacional, ressalta-se a importância do uso do instrumento digital para garantir a validade das medidas. Deve-se destacar que a pressão sustentada, na diretriz internacional, é definida como a medida da média da pressão máxima sustentada por 1 segundo (pressão média máxima). Para a diretriz nacional, a pressão sustentada é definida como a manutenção da pressão inspiratória e expiratória máxima após o primeiro segundo (pressão de platô), assumindo valor inferior ao pico de pressão mais elevado (pressão de pico). No Brasil, os pesquisadores Neder et al. ${ }^{8}$, Costa et al. ${ }^{9}$, Simões et al. ${ }^{10}$ e Pessoa et al. ${ }^{5}$ propuseram equações de predição para PRM, sendo que os três últimos autores elaboraram suas equações após a publicação das diretrizes.

O estudo de Neder et al. ${ }^{8}$, em relação às recomendações das diretrizes, não referiu o diâmetro do orifício de fuga, que serve para evitar o aumento da pressão intraoral causado pela contração dos músculos bucinadores. Se ausente ou menor que $1 \mathrm{~mm}$, o valor da PImáx pode ser superestimado ${ }^{2}$. No presente estudo, ao observar os valores médios preditos por Neder et al. ${ }^{8}$ para a PImáx, constata-se haver uma superestimação quando comparado com os demais autores. Essa constatação é corroborada pelo estudo de Leal et al. ${ }^{19}$, que também verificou valores superestimados nas equações de Neder et al. ${ }^{8}$ ao comparar com as de Black e Hyatt ${ }^{20}$ e Harink-Khan ${ }^{21}$.

Em contrapartida, verificou-se que os valores preditos por Simões et al. ${ }^{10}$ foram subestimados em relação aos demais - levando em conta que esse estudo foi realizado com uma amostra de indivíduos sedentários, o que deixa supor que os baixos valores preditos se devem ao sedentarismo, que pode influenciar as PRM.
Pessoa et al. ${ }^{5}$ objetivaram estabelecer equações de referência para as PRM conforme recomendações das diretrizes. Foram recrutados brasileiros saudáveis, sem alteração cognitiva constatada pelo MEEM; foi o único estudo brasileiro a usar o MVC digital (NEPEBLabCare/ UFMG) com transdutor de pressão com intervalo operacional de $\pm 500 \mathrm{cmH}_{2} \mathrm{O}$.

Nota-se que o estudo de Neder et al. ${ }^{8}$, embora realizado antes da publicação das referidas diretrizes, apresentou um alto rigor no controle das variáveis biológicas e contou com aspectos metodológicos bem definidos, similar ao estudo de Pessoa et al. ${ }^{5}$. Verificou-se que apenas Neder et al. ${ }^{8} \mathrm{e}$ Pessoa et al. ${ }^{5}$ apresentaram diferenças significativas entre os valores obtidos e os preditos para a PImáx. Ressaltase que a amostra do presente estudo foi constituída por indivíduos com fraqueza muscular $\left(\mathrm{PImáx}>-80 \mathrm{cmH}_{2} \mathrm{O}\right)^{1,2}$. Dessa forma infere-se que ambas as equações são seguras para detectar fraqueza muscular inspiratória.

Uma recente revisão sistemática com meta-análise ${ }^{4}$ concluiu que variáveis biológicas e variáveis metodológicas influenciam nos valores preditos para a PImáx. Essa premissa respalda a discrepância entre os valores das PImáx encontradas entre os autores brasileiros verificadas no presente estudo.

A variação entre os resultados das equações de predição dos autores brasileiros foi observada na análise de BlandAltman. Os valores das equações de predição para a PImáx demonstraram pouca concordância, como pode ser exemplificado pelos valores de PImáx propostos por Neder et al. ${ }^{8}$ e Simões et al. ${ }^{10}$. O Bias indica o quanto as diferenças entre os valores preditos se afastam do valor zero. Além disso, os limites de concordância de $95 \%$ ou o erro, que consiste na dispersão dos pontos das diferenças ao redor da média, foram inadequados - indicando que há uma maior diferença ou uma menor concordância entre os valores preditos.

Constata-se, também, que, embora a equação de Pessoa et al. ${ }^{5}$ tenha sido criada com o MVC digital, ela pode ser utilizada na prática clínica quando o uso do equipamento analógico for empregado - uma vez que não houve diferença significativa entre os resultados das equações de predição propostas por Neder et al..$^{8}$ e Pessoa et al. ${ }^{5}$

Diante do exposto, sugere-se que a escolha da equação para ser usada na prática clínica leve em consideração: equações que não superestimam ou subestimam os valores preditos; similaridade com as características biológicas da amostra referente à criação dos valores de referência (sedentarismo, por exemplo) e equações criadas com rigor metodológico proposto pelas diretrizes para as PRM. 
Aponta-se como limitação deste estudo um baixo número amostral ( $\mathrm{n}=18)$, embora a amostragem estivesse acima do cálculo amostral realizado inicialmente $(n=7)$. É importante ressaltar que o poder do estudo foi calculado posteriormente por meio do software $\mathrm{G}^{*}$ Power 3.1, que demonstrou valor de 0,99 com um tamanho de efeito moderado ${ }^{22}$.

\section{CONCLUSÃO}

Os valores obtidos e preditos para a PImáx não foram semelhantes entre os autores; apenas Neder et al. ${ }^{8}$ e Pessoa et al. ${ }^{5}$ detectaram diferença significativa entre os valores obtidos e preditos pelas equações. Neder et al. ${ }^{8}$ e Pessoa et al..$^{5}$ apresentaram maior concordância nos valores de PImáx preditos, embora os valores entre as quatro equações brasileiras apresentassem diferença significativa.

\section{REFERÊNCIAS}

1. ATS/ERS. Statement on respiratory muscle testing. Am J Respir Crit Care Med. 2002;166(4):518-624. doi: 10.1164/rccm.166.4.518

2. Souza, RB. Pressões respiratórias estáticas máximas. J Pneumol. 2002;28(Supl 3):155-65. [cited 2021 Mar 4]. Available from:http:// www.jornaldepneumologia.com.br/PDF/Suple_137_45_88\%20 Press \% C $3 \%$ B 5 es $\% 2$ orespirat \% C 3\% B 3 rias $\% 20$ est\%C3\%A1ticas\%20m\%C3\%A1ximas.pdf

3. Ringqvist T. The ventilatory capacity in healthy subjects: an analysis of causal factors with special reference to the respiratory forces. Scand J Clin Lab Invest Suppl. 1966;88:5179. [cited 2021 Mar 4]. Available from: https://europepmc. org/abstract/med/4283858

4. Pessoa IMBS, Parreira VF, Fregonezi GAF, Sheel AW, Chung F, Reid WD. Reference values for maximal inspiratory pressure: a systematic review. Can Respir J. 2014;21(1):43-50. doi: 10.1155/2014/982374

5. Pessoa IMBS, Neto MH, Montemezzo D, Silva LAM, Andrade AD, Parreira VF. Predictive equations for respiratory muscle strength according to international and Brazilian guidelines. Braz J Phys Ther. 2014;18(5):410-8. doi: 10.1590/bjpt-rbf.2014.0044

6. Mehta S. Neuromuscular disease causing acute respiratory failure. Respir Care. 2006; 51(9):1016- 21. [cited 2021 Mar 4]. Available from: https://pubmed.ncbi.nlm.nih.gov/16934165/

7. Meyer FJ, Borst MM, Zugck C, Kirschke A, Schellberg D, Kübler W, et al. Respiratory muscle dysfunction in congestive heart failure: clinical correlation and prognostic significance. Circulation. 2001;103(17):2153-8. doi: 10.1161/01.CIR.103.17.2153

8. Neder JA, Andreoni S, Lerario MC, Nery LE. Reference values for lung function tests: maximal respiratory pressures and voluntary ventilation II. Braz J Med Biol Res. 1999;32(6):719-27. doi: 10.1590/S0100-879X1999000600007
9. Costa D, Gonçalves HA, Lima LP, Ike D, Cancelliero KM, Montebelo MI. New reference values for maximal respiratory pressures in the Brazilian population. J Bras Pneumol. 2010;36(3):306-12. doi:10.1590/S1806-37132010000300007

10. Simões RP, Deus APL, Auad MA, Dionísio J, Mazzoneto M, Silva AB. Maximal respiratory pressure in healthy 20 to 89 year-old sedentary individuals of central Sao Paulo State. Rev Bras Fisioter. 2010;14(1):60-7. doi: 10.1590/S1413-35552010000100010

11. Godoy-Matos AF, Oliveira J, Guedes EP, Carraro L, Lopes AC, Mancini MC, et al. In: Diretrizes brasileiras de obesidade 2009/2010 / ABESO - Associação Brasileira para o Estudo da Obesidade e da Síndrome Metabólica. 3rd ed. Itapevi: AC Farmacêutica; 2009 [cited 2021 Feb 20]. p. 7-25. Available from: http://www.saude. df.gov.br/wp-conteudo/uploads/2018/08/2009_DIRETRIZES_ BRASILEIRAS_DE_OBESIDADE.pdf

12. Guedes GP, Barbosa YRA, Holanda G. Correlação entre força muscular respiratória e tempo de internação pós-operatório. Fisioter Mov. 2009;22(4):605-14. [cited 2021 Mar 4]. Available from: https://periodicos.pucpr.br/index.php/fisio/article/ view/19521

13. Brucki SM, Nitrini R, Caramelli P, Bertolucci PHF, Okamoto IH. Suggestions for utilization of the mini-mental state examination in Brazil. Arq Neuropsiquiatr. 2003;61(3B):777-81. doi: 10.1590/ S0004-282X2003000500014

14. Montemezzo D, Vieira DSR, Tierra-Criollo CJ, Britto RR, Velloso $M$, Parreira VF. Influence of 4 interfaces in the assessment of maximal respiratory pressures. Respir Care. 2012;57(3):392-38. doi: 10.4187/respcare.01078

15. Enright PL, Adams AB, Boyle PJ, Sherriel DL. Spirometry and maximal respiratory pressure references from healthy Minnesota 65- to 85-year-old women and men. Chest. 1995;108(3):663-9. doi: 10.1378/chest.108.3.663.

16. Enright PL, Kronmal RA, Manolio TA, Schenker MB, Hyatt RE. Respiratory muscle strength in the elderly: correlates and reference values: Cardiovascular Health Study Research Group. Am J Respir Crit Care Med. 1994;149(2 Pt 1):430-8. doi: 10.1164/ ajrccm.149.2.8306041

17. Vincken W, Ghezzo H, Cosio MG. Maximal static respiratory pressures in adults: normal values and their relationship to determinants of respiratory function. Bull Eur Physiopathol Respir. 1987;23(5):435-9. [cited 2021 Mar 4]. Available from: https://europepmc.org/article/med/3450325

18. Johan A, Chann CC, Chia HP, Chan OY, Wang YT. Maximal respiratory pressures in adult Chinese, Malays and Indians. Eur Respir J. 1997;10(12):2825-28. doi: 10.1183/09031936.97.10122825

19. Leal AH, Hamasaki TA, Jamami M, Lorenzo VAPD, Pessoa BV. Comparação entre valores de força muscular respiratória medidos e previstos por diferentes equações. Rev Bras Fisioter. 2007;14(3):25-30. doi: 10.1590/fpusp.v14i3.76090

20. Black LF, Hyatt RE. Maximal respiratory pressures: normal values and relationship to age and sex. Am Rev Respir Dis. 1969;99(5):696-702. doi: 10.1164/arrd.1969.99.5.696

21. Harik-Khan RI, Wise RA, Fozard JL. Determinants of maximal inspiratory pressure: the Baltimore longitudinal study of aging. Am J Respir Crit Care Med. 1998;158(5 Pt 1):1459-64. doi: 10.1164/ ajrccm.158.5.9712006

22. Cohen J. Statistical power analysis for the behavioral sciences. 2nd ed. Hillsdale, NJ: Erlbaum; 1988. 